the pathophysiology of migraine, ${ }^{5}$ and dysfunction here may also be responsible for the impairment of consciousness.

In the present case, loss of consciousness soon after head injury very reasonably led to the diagnosis of acute extradural haemorrhage. In other cases, such features as drowsiness, confusion and hemiparesis following head injury have also led to admission to neurosurgical units. ${ }^{2}$ Such patients were formerly investigated by angiography, which often caused neurological deterioration, ${ }^{5}$ and findings suggestive of an avascular space occupying lesion (thought to be due to oedema) led in some cases to inappropriate surgical exploration. ${ }^{2}$

Where coma or focal neurological dysfunction follow a mild head injury, and CT is negative, the diagnosis of trauma-induced migraine should be considered.

RODERICK DUNCAN

ALASTAIR JENKINS

Institute of Neurological Sciences,

Southern General Hospital

Govan Road, Glasgow

G5I $4 T F, U K$

References

1 Haas DC, Lourie H. Trauma-triggered migraine: an explanation for common neurological attacks after mild head injury. J Neurosurg 1988;68:181-8.

2 Whitty CWM. Handbook of Clinical Neurology, Vol. 4. Amsterdam: Elsevier Science Publishers, 1986:148.

3 Lazorthes Y, Van Hong N, Lazorthes G. Les aggravations secondaire precoces survenant apres les traumatismes cranio-cerebraux de l'enfant. Neurochirurgie, 1969;15:19-26.

4 Fitzsimmons RB, Wolfenden WH. Migraine coma. Brain 1985; 108:555-77.

5 Lance JW, Adams RW, Lambert GA. Bulbocortical pathways and their possible relevance to migraine and epilepsy. Functional Neurology, 1986;1:357-61.

Accepted 2 February 1989

\section{Relapsing polyneuritis following classic Miller Fisher syndrome}

Sir: The Miller Fisher syndrome is a well described form of acute inflammatory polyneuropathy, a variant of the Guillain-Barré syndrome. Initially reported by Fisher in $1956,{ }^{1}$ this disease is characterised by the acute onset of ataxia, areflexia and ophthalmoplegia often preceded by a viral or other illness. Despite the alarming presentation, the disease usually has a benign outcome with a spontaneous and complete recovery within weeks. We report a case of classic Miller Fisher Syndrome followed by a bout of relapsing polyneuritis 8 weeks later.

A 21 year old white female student presented with complaints of facial paraesthesias, diplopia, generalised weakness, and a gait disturbance described as a "loss of balance sense."

Physical examination of heart, lung and abdomen was normal. Neurological examination revealed an ophthalmoplegia involving all extraocular muscles with minimal vertical gaze preserved bilaterally. Bilateral ptosis was noted, with no nystagmus. Pupils were reactive to light and accommodation. Deep tendon reflexes were slight in the upper extremities and absent in the lower extremities. Her upper and lower extremity strength was intact bilaterally, as was sensory perception. Ataxia was present, with a wide based, unsteady gait.

Lumbar puncture revealed a glucose of 58 $\mathrm{mg} / 100 \mathrm{ml}$, protein of $72 \mathrm{mg} / 100 \mathrm{ml}$, and a cell count of $10 / \mathrm{mm}^{3} \mathrm{RBC}$ and $1 / \mathrm{mm}^{3} \mathrm{WBC}$. Nerve conduction and EMG studies were performed. Skin temperature was within the normal range $\left(31^{\circ} \mathrm{C}\right)$. Peroneal nerve motor velocity was $44 \mathrm{~m} / \mathrm{s}$. F wave conductions were slow at $35 \mathrm{~mm} / \mathrm{s}$. Sural responses were absent despite averaging. This was consistent with a mild demyelinating neuropathy.

The patient was diagnosed as suffering from Miller Fisher syndrome and was observed in the intensive care unit. Over the next several days, the patient experienced progressive leg and arm paraesthesias, without a deficit in limb strength. Slowly, the patient improved, and was discharged 10 days after admission with minimal ptosis, paraesthesias, facial weakness and ataxia.

The patient was seen one month later where she was found to be walking easily and feeling stronger. There was full ocular motility, with minimal ptosis of the left eye and mild right sided facial weakness. Sensory testing revealed minimally diminished vibration and position sense of hands and fingers with mildly diminished pin sensation in a stocking distribution. The gait was normal. Deep tendon reflexes were absent.

One week later she developed an exacerbation of her neurological symptoms. Ambulatory skills declined with development of foot and hand paraesthesias. On reexamination the patient was noted to have no position sense with manual testing of her hands and feet or any distal vibratory sense. Strength was good and her extraocular muscles remained intact. She had no exacerbation of her facial weakness or ptosis. Her dramatic clinical decline was consistent with a disabling relapsing polyneuropathy, primarily sensory in nature. She was the started on a two week regimen of orat prednisone.

After one week of steroid therapy, th patient reported a dramatic improvemem in her symptomatology. Unsteadines imbalance and diminished position were lesf noticeable. It was felt that she was entering remission stage of her neuropathy.

Physical examination three months aft her initial diagnosis revealed persistence of the absent deep tendon reflexes. The facial weakness and ptosis, significant at the onse्ध of her illness, were no longer apparenf Extraocular muscle movement was withog limitation. Tactile sensation was norma although position and vibration were st mildly impaired. She was able to walk witio out assistance and anticipated returning her activities of normal living.

The Miller Fisher syndrome is a rare for $\overrightarrow{\mathbb{T}}$ of acquired demyelinating neuropathy.' variant of Guillain-Barré syndrome, th monophasic illness develops acutely over few days. The ataxia is often severe and debilitating. Symmetrical ocular palsieg occur with greater involvement of externab musculature. Limb involvement is mining or absent, although motor weakness क्री been known to occur. Patients uslally recover without specific treatment overgh course of several weeks after a relatioely benign but frightening illness.

Our patient exhibited all the features syndrome. What distinguished our case the others was the development of recuning polyneuritis complicating the initial \$af nosis of Miller Fisher syndrome. We did not expect her to relapse after her rather benig initial course. Interestingly, during the time of the relapse, the patient did not exhibit an exacerbation of her ophthalmoplegia facial weakness. Her main problem was sensory with loss of position and vibrator sense of her extremities, as well as pin touch A dramatic improvement was noted after the introduction of corticosteroid therapy.

A review of the literature has yielded similar but not identical cases. Donaghy $\overline{\vec{e}}$ $a l^{2}$ reported three cases of ocular palsy preceding the development of chronic polg neuritis by an interval of two to ten week Two patients with ophthalmoparesis ang recurrent sensory neuropathy were reported by Kaplan. ${ }^{3}$ Chalmers and Miller in $198 \overline{6}$ reported a patient with a 2 year history of chronic progressive inflammatory poly neuropathy with a subacute exacerbation. that included ataxia, areflexia, ophthatmoparesis and ptosis. Schapira ${ }^{5}$ reported $\Re$ patient with a relapsing neurological deficis He initially manifested the Miller Fishe्c 
syndrome with Guillain-Barré features (limb weakness and distal paresthesias), and during a relapse one year later, exhibited purely Miller Fisher features.

Our patient had a classic manifestation of Miller Fisher syndrome with ataxia, areflexia and ophthalmoplegia followed by a recurring polyneuropathy eight weeks later. We believe this is the first reported case of this association. Most often the course of Miller Fisher syndrome is one of spontaneous recovery. This patient reinforces the need for continued observation of these patients, even after initial improvement of their neurological deficit.

DR KAISER-SMITH Department of Medicine, Section of General Internal Medicine, University of Medicine and Dentistry of New

Jersey,

School of Osteopathic Medicine, 301 S. Central Plaza, Suite 3100,

Stratford, NJ 08084

DR BARONE

Department of Medicine, Section of Neurology,

University of Medicine and Dentistry of New

Jersey,

School of Osteopathic Medicine

PO Box 330

Voorhees, NJ 08043, USA

\section{References}

1 Fisher CM. An unusual variant of acute idiopathic polyneuritis (syndrome of ophthalmoplegia, ataxia and areflexia). $N$ Engl $J$ Med 1956;255:57-65.

2 Donaghy H, Earl CJ. Ocular palsy preceding chronic relapsing polyneuropathy by several weeks. Ann Neurol 1985;17:49-50.

3 Kaplan JG, Schaumburg HH, Sumner A. Relapsing ophthalmoparesis-sensory neuropathy syndrome. Neurology 1985; 35:595-6.

4 Chalmers AC, Miller RG. Chronic inflammatory polyradiculoneuropathy with ophthalmoplegia. J Clin Neuro-Ophthalmol 1986;6:166-8.

5 Schapira AHV, Thomas PKA. Case of recurrent idiopathic ophthalmoplegic neuropathy (Miller Fisher Syndrome). $J$ Neurol Neurosurg Psychiatry 1986;49:463.

\section{Spontaneous haemorrhage in craniopharyn-} gioma

Sir: While chemical meningitis caused by the extravasation of oily cystic fluid from a ruptured craniopharyngioma is known, it usually occurs after surgery. Spontaneous rupture is more rare' and proven haemorrhage has not been documented in this tumour. We describe a patient who presented with subarachnoid haemorrhage secondary to haemorrhage in a craniopharyngioma.

A 59 year old housewife, who had been suffering from sciatic pain and mild headache for two months developed severe headache and nausea on the day before admission. She had no contributory previous medical or family history. On examination the patient was alert, cooperative but was in some distress. The detected abnormalities were mild fever and pain bilaterally upon straight leg raising. Her neck was supple.

Lumbar puncture yielded deeply xanthochromic, turbid cerebrospinal fluid (CSF) with the opening pressure of $22 \mathrm{~cm} \mathrm{H}_{2} \mathrm{O}$. Other than the uncounted erythrocytes, the cell number of the CSF was 250 per $\mathrm{mm}^{3}$ with $59 \%$ polymorphonuclear leukocytes, $38 \%$ lymphocytes, and $3 \%$ monocytoid cells; the protein was $125 \mathrm{mg} / \mathrm{dl}$, and the glucose 38 $\mathrm{mg} / \mathrm{dl}$. There were no detectable cholesterol crystals. The CSF cytology was negative. CT revealed a high density suprasellar mass (fig A) which was diffusely enhanced by contrast medium. Plain radiographs of the skull demonstrated calcification in the suprasellar region corresponding to the mass in CT. The cerebral angiogram was unremarkable. The tentative diagnosis was a haemorrhage either into a craniopharyngioma or a pituitary adenoma. Laboratory examination of the peripheral blood was unremarkable except for the mildly elevated prolactin level.

Repeat CT without contrast medium revealed a gradual decrease in the density of the juxtasellar portion of the mass (fig B).

Two months after admission, subtotal
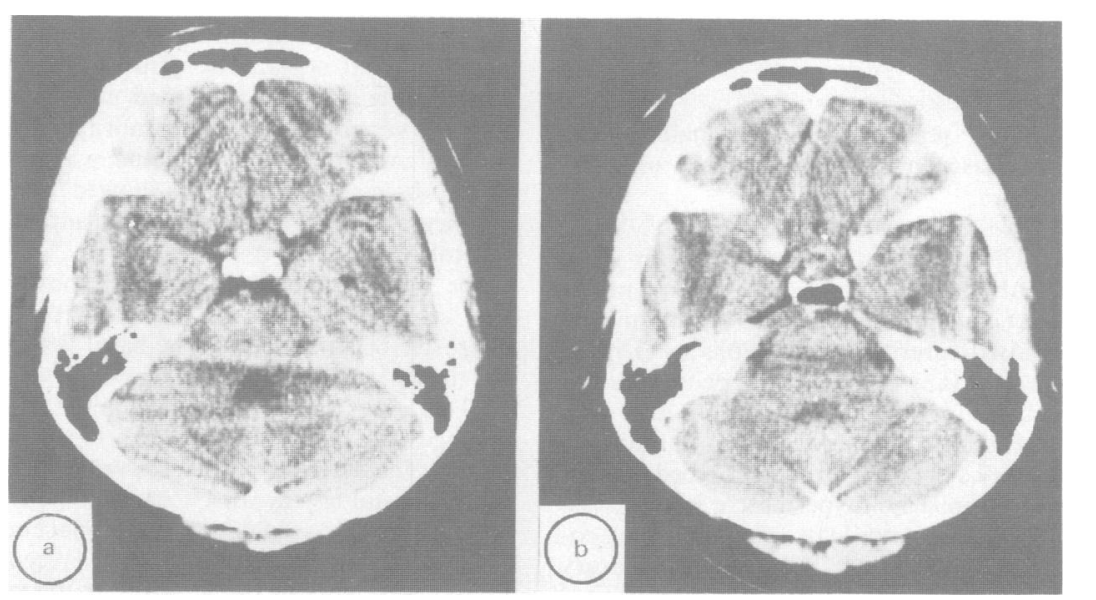

Fig CT without contrast medium, showing a juxtasellar high density mass (A) I day and (B) 42 days after the onset of symptom.

removal of the suprasellar tumour was performed via the right fronto-tempora approach. The arachnoid membrane was brownish and thick. Behind the optic chiasm was a partly dark coloured, partly calcified mass which did not yield any fluid. Histological examination of the specimen demonstrated typical craniopharyngioma consisting of islands of epidermoid tissue in the adamanitinomatous pattern, squamous cells, keratinisation, calcification, and microcyst formation. In addition, there were blood clots and numerous haemosiderinladen macrophages. Some of the blood vessel walls in the tumour and the surrounding connective tissue stroma showed hyaline or fibrous thickening. The postoperative course was uneventful, except for transient diabetes insipidus.

The clinical manifestations of craniopharyngioma include visual field deficit, hypopituitarism, increased intracranial pressure caused by compression of the surrounding structures and chemical meningitis due to extravasation of cystic fluid. However, haemorrhage has not been confidently proved to occur in craniopharyngioma as it has, rarely, in pituitary adenoma. ${ }^{2}$

As in the present case, the CT density of some of craniopharyngiomas has beer reported to vary with time. ${ }^{34}$ In such a cased the CT change was attributed to the protein concentration, not to haemorrhage, in the cyst. $^{3}$ In another case haemosiderin deposio tion was found in the connective tissue stroma of the cyst wall. The authors sugges ted haemorrhage in the tumour although there had been no sudden episode of clinical deterioration or bloody cyst fluid at surgery. ${ }^{4}$ The CSF and preoperative CT findings of 